\title{
The arterial blood pressure associated with terminal cardiovascular collapse in critically ill patients: a retrospective cohort study
}

\author{
Andreas Brunauer ${ }^{1}$, Andreas Koköfer ${ }^{1}$, Otgon Bataar ${ }^{2}, \| s e$ Gradwohl-Matis ${ }^{1}$, Daniel Dankl ${ }^{1}$ and Martin W Dünser ${ }^{1 *}$
}

\begin{abstract}
Introduction: Liberal and overaggressive use of vasopressors during the initial period of shock resuscitation may compromise organ perfusion and worsen outcome. When transiently applying the concept of permissive hypotension, it would be helpful to know at which arterial blood pressure terminal cardiovascular collapse occurs.

Methods: In this retrospective cohort study, we aimed to identify the arterial blood pressure associated with terminal cardiovascular collapse in 140 patients who died in the intensive care unit while being invasively monitored. Demographic data, co-morbid conditions and clinical data at admission and during the 24 hours before and at the time of terminal cardiovascular collapse were collected. The systolic, mean and diastolic arterial blood pressures immediately before terminal cardiovascular collapse were documented. Terminal cardiovascular collapse was defined as an abrupt ( $<5$ minutes) and exponential decrease in heart rate $(>50 \%$ compared to preceding values) followed by cardiac arrest.
\end{abstract}

Results: The mean \pm standard deviation (SD) values of the systolic, mean and diastolic arterial blood pressures associated with terminal cardiovascular collapse were $47 \pm 12 \mathrm{mmHg}, 35 \pm 11 \mathrm{mmHg}$ and $29 \pm 9 \mathrm{mmHg}$, respectively. Patients with congestive heart failure ( $39 \pm 13 \mathrm{mmHg}$ versus $34 \pm 10 \mathrm{mmHg} ; P=0.04)$, left main stem stenosis ( $39 \pm 11 \mathrm{mmHg}$ versus $34 \pm 11 \mathrm{mmHg} ; P=0.03)$ or acute right heart failure $(39 \pm 13 \mathrm{mmHg}$ versus $34 \pm 10 \mathrm{mmHg} ; P=0.03)$ had higher arterial blood pressures than patients without these risk factors. Patients with severe valvular aortic stenosis had the highest arterial blood pressures associated with terminal cardiovascular collapse (systolic, $60 \pm 20 \mathrm{mmHg}$; mean, $46 \pm 12 \mathrm{mmHg}$; diastolic, $36 \pm 10 \mathrm{mmHg}$ ), but this difference was not significant. Patients with sepsis and patients exposed to sedatives or opioids during the terminal phase exhibited lower arterial blood pressures than patients without sepsis or administration of such drugs.

Conclusions: The arterial blood pressure associated with terminal cardiovascular collapse in critically ill patients was very low and varied with individual co-morbid conditions (for example, congestive heart failure, left main stem stenosis, severe valvular aortic stenosis, acute right heart failure), drug exposure (for example, sedatives or opioids) and the type of acute illness (for example, sepsis).

\section{Introduction}

In patients with shock, it is imperative to reestablish adequate systemic blood flow and reverse tissue hypoperfusion [1]. This can typically be achieved by the administration of fluids and/or inotropes [1,2]. In severe arterial hypotension, however, the treatment response to these interventions may

\footnotetext{
* Correspondence: m.duenser@salk.at

'Department of Anesthesiology, Perioperative Care and Intensive Care Medicine, University Hospital Salzburg and Paracelsus Private Medical University, Müllner Hauptstrasse 48, 5020 Salzburg, Austria

Full list of author information is available at the end of the article
}

be too slow to rapidly increase arterial blood pressure levels to currently recommended levels (for example, a mean arterial blood pressure $\geq 65 \mathrm{mmHg}[3,4]$ ), despite reversal of the underlying pathology (for example, replenishment of hypovolemia, inotropic therapy). This is the reason why vasopressor drugs, such as norepinephrine, are commonly started liberally to avoid cardiovascular collapse due to ongoing arterial hypotension. Recent data, however, suggest that liberal use of vasoactive agents during the first hour may adversely affect mortality due to shock [5], particularly when systemic hypoperfusion is present, such as during 
hemorrhage [6]. Similarly, Subramanian et al. showed that restrictive vasopressor therapy during the first hours of shock can lead to improved tissue perfusion and less organ dysfunction, putatively by avoiding vasopressor-induced aggravation of systemic vasoconstriction and aggravation of tissue hypoperfusion [7]. Even marked delays in initiation of vasopressor therapy were associated with only a small increase in the risk of death in 8,640 patients with septic shock [8]. Because a strategy of restrictive vasopressor use during the early phase of shock implies that a certain degree of arterial hypotension is transiently tolerated to buy time so that definite hemorrhage control can be achieved (for example, in traumatic-hemorrhagic shock) [9] or ongoing fluid resuscitation and inotropic therapy can restore systemic blood flow (for example, in septic shock) [10]. When implementing such a concept of permissive hypotension with a primary focus on reversal of systemic hypoperfusion during early shock resuscitation, it would be helpful to know the range of arterial hypotension levels within which cardiovascular collapse occurs. Identification of these levels of arterial blood pressure, together with a certain safety margin, could help to guide emergent use of vasopressor drugs and avoid overaggressive use of vasoconstrictors in patients with systemic hypoperfusion [10,11].

In this retrospective cohort study, we aimed to identify the arterial blood pressure associated with terminal cardiovascular collapse in 140 patients who died in the intensive care unit (ICU) while being invasively monitored.

\section{Materials and methods}

This study was designed as a retrospective cohort analysis. It included patients admitted to a 37-bed medicalsurgical ICU of a tertiary university teaching hospital between July 2011 and April 2013. The study protocol was evaluated by the local ethics committee (Ethikkomission für das Bundesland Salzburg; 415-EP/73/203-2013), which waived the requirement for written informed consent because of the retrospective study design.

\section{Study patients and data collection}

All critically ill patients who were invasively monitored and died in the ICU during the observation period were eligible for enrollment. The exclusion criteria were age younger than 18 years, pregnancy, organ donation following brain(stem) death, immediate death after withdrawal of ventricular assist device support and absence of continuous electrocardiographic or invasive arterial blood pressure measurements.

All study variables were extracted from the institutional electronic patient data management system (MetaVision; IMDSoft, Tel Aviv, Israel). This system prospectively collects demographic data and patient characteristics; hemodynamic and other vital parameters are collected at one minute intervals. The system uses median filtering at 1-minute intervals, which is an effective nonlinear, digital filtering process to eliminate artefacts due to a signal [12]. Data regarding drugs and fluids administered are entered manually into the database.

The following study variables were extracted from the database: age, sex, body mass index, premorbid conditions (known at ICU admission and/or diagnosed at autopsy), admission diagnosis, Simplified Acute Physiology Score (SAPS) II [13] and SAPS III [14] scores, end-of-life decisions, time from terminal cardiovascular collapse until death, cause of death, length of ICU stay and (wherever available) autopsy results. During the 24 hours before the terminal cardiovascular collapse, we documented the Sequential Organ Failure Assessment (SOFA) score [15], serum creatine kinase MB levels, as well as the presence and focus of sepsis [16], acute right heart failure (defined as low cardiac output due to increased pulmonary arterial pressure or decreased right ventricular contractility diagnosed by echocardiography or pulmonary artery catheter measurements) or shock (defined as need for catecholamine infusions to maintain tissue perfusion). At the time of the terminal cardiovascular collapse (see definition below), we collected the data for plethysmographic oxygen saturation; body temperature; heart rate; systolic, mean and diastolic arterial blood pressures (zeroing at the midchest level); central venous pressure; doses of catecholamines, vasopressin and/or levosimendan; exposure to sedative drugs and/or opioids; and last measurements of arterial lactate, base deficit, hemoglobin and partial arterial carbon dioxide tension.

\section{Determination of the arterial blood pressure associated with terminal cardiovascular collapse}

The arterial blood pressure associated with terminal cardiovascular collapse was defined as the systolic, mean and diastolic arterial blood pressures immediately (within 1 minute) before terminal cardiovascular collapse occurred. Terminal cardiovascular collapse was defined as an abrupt ( $<5$ minutes) and exponential decrease in heart rate ( $>50 \%$ compared to preceding values) followed by cardiac arrest (Figure 1). Patients in whom the drop in heart rate preceded the fall in arterial blood pressure were considered to have rhythmologic pathologies and were excluded from the analysis.

\section{Study endpoints}

The primary endpoint of the study was to identify the systolic, mean and diastolic arterial blood pressure levels associated with terminal cardiovascular collapse in this study population. Secondary endpoints were to determine and compare these arterial blood pressure levels within and between the following subgroups: age $>65$ years, age $>75$ years, cardiac surgery, chronic arterial hypertension, congestive heart failure, coronary artery disease, 


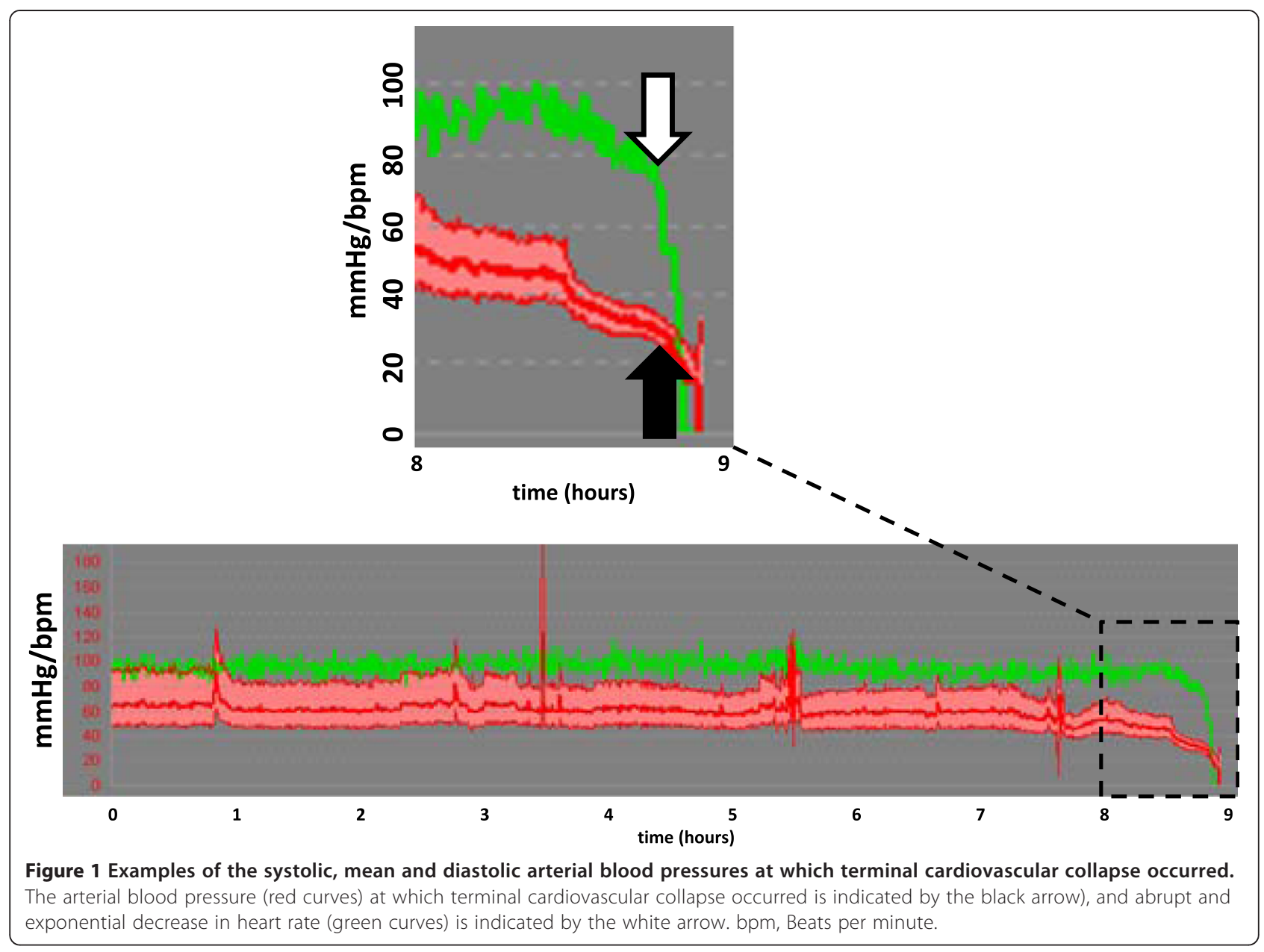

diabetes mellitus, left main stem stenosis or equivalent, peripheral arterial occlusive disease, severe valvular aortic stenosis (grade 3 or 4), acute right heart failure, sepsis and shock. In addition, we analyzed whether the arterial blood pressure at terminal cardiovascular collapse was correlated with any of the following simultaneously measured variables: age, SOFA score, hemoglobin, partial arterial carbon dioxide tension, plethysmographic oxygen saturation, body temperature and exposition to sedative drugs and/or opioids.

\section{Statistical analysis}

All statistical analyses were performed using the PASW Statistics 18 software package (SPSS, Chicago, IL, USA). Following plausibility testing of the dataset, the normality distribution was checked by using the Kolmogorov-Smirnov test and was approximately fulfilled by all variables. Descriptive methods were used to present patient characteristics and the arterial blood pressures associated with terminal cardiovascular collapse in the study population and subgroups. The unpaired Student's $t$-test was used to compare these arterial blood pressure levels between subgroups.
Bivariate correlations calculated by applying the Pearson correlation coefficient were used to evaluate the relationship between the arterial blood pressure associated with terminal cardiovascular collapse and predefined cofactors. $P$-values $<0.05$ were considered to indicate statistical significance. All parameters are presented as mean values $\pm S D$, if not indicated otherwise.

\section{Results}

Two hundred thirteen ICU patients died during the observation period. Of these, no one was aged $<18$ years or pregnant, but electrocardiographic or invasive blood pressure measurements were not recorded in 22 patients. Nine patients died after organ donation following brain(stem) death, and five died after withdrawal of venoarterial extracorporeal membrane oxygenation therapy. Of the remaining 177 patients, 27 had rhythmological pathologies in the terminal phase. In ten patients, the arterial blood pressure at terminal cardiovascular collapse could not be determined. Following exclusion of these patients, 140 patients were included in the final analysis (Tables 1 and 2). In 120 patients (85.7\%), end-of-life decisions were 
Table 1 Characteristics of the study population

\begin{tabular}{ll}
\hline Characteristics & Patient data $(\mathbf{N = 1 4 0 )}$ \\
\hline Age (yr) & $72.8 \pm 13.2$ \\
Male sex $(n(\%))$ & $87(62.1)$ \\
Body mass index $\left(\mathrm{kg} / \mathrm{m}^{2}\right)$ & $26.1 \pm 5.5$ \\
Comorbid conditions $(n(\%))$ & \\
$\quad$ Chronic arterial hypertension & $78(55.7)$ \\
$\quad$ Coronary artery disease & $57(40.7)$ \\
Congestive heart failure & $27(19.3)$ \\
Severe aortic stenosis & $3(2.1)$ \\
Chronic renal failure & $26(18.6)$ \\
Diabetes mellitus & $26(18.6)$ \\
Peripheral arterial occlusive disease & $15(10.7)$ \\
Admission diagnosis ( $n$ (\%)) & \\
$\quad$ Post-cardiac arrest & $26(18.6)$ \\
Shock of any origin & $22(15.7)$ \\
Abdominal disease & $21(15)$ \\
Respiratory insufficiency & $20(14.3)$ \\
Trauma & $12(8.6)$ \\
Post-cardiac surgery & $10(7.1)$ \\
Gastrointestinal hemorrhage & $7(5)$ \\
Miscellaneous & $22(15.6)$ \\
SAPS III ${ }^{a}$ (points) & $53 \pm 20$ \\
Antensive care unit length of stay (days) & $73 \pm 18$ \\
\hline
\end{tabular}

${ }^{a}$ SAPS, Simplified Acute Physiology Score. Data are presented as mean values $\pm S D$, if not otherwise indicated.

made to withhold or withdraw invasive organ support. At the time of terminal cardiovascular collapse, 113 patients (80.7\%) were receiving sedatives, opioids or both.

The intraobserver and interobserver agreement for identification of the arterial blood pressure associated with terminal cardiovascular collapse were $94 \%$ and $88 \%$, respectively. The systolic, mean and diastolic arterial blood pressures (mean $\pm \mathrm{SD}$ ) associated with terminal cardiovascular collapse were $47 \pm 12 \mathrm{mmHg}, 35 \pm 11 \mathrm{mmHg}$ and $29 \pm 9 \mathrm{mmHg}$, respectively (Table 3 ). The time from cardiovascular collapse to death was $31 \pm 30$ minutes. Patients with congestive heart failure, left main stem stenosis or acute right heart failure had higher mean arterial blood pressures at terminal cardiovascular collapse than patients without these risk factors. Among the patients with congestive heart failure, $77.8 \%$ had coronary artery disease and $44.4 \%$ had left main stem stenosis. Patients with severe valvular aortic stenosis had the highest arterial blood pressure at terminal cardiovascular collapse, but this difference missed the significance level (Table 3, Figure 2). Patients with sepsis during the 24 hours before terminal
Table 2 Clinical data 24 hours before and at the time of terminal cardiovascular collapse, as well as causes of death

\begin{tabular}{|c|c|}
\hline & Clinical data \\
\hline \multicolumn{2}{|l|}{24 hours before terminal cardiovascular collapse } \\
\hline Sepsis $(n(\%))$ & $34(24.3)$ \\
\hline \multicolumn{2}{|l|}{ Sepsis focus ( $n(\%))$} \\
\hline Abdomen & $9(6.4)$ \\
\hline Soft tissue/joints & $7(5)$ \\
\hline Lungs & $3(2.1)$ \\
\hline Primary bacteremia & $2(1.4)$ \\
\hline Miscellaneous & $18(9.3)$ \\
\hline Shock (n (\%)) & $87(62.1)$ \\
\hline Acute right heart failure $(n(\%))$ & $22(15.7)$ \\
\hline Sequential Organ Failure Assessment score (points) & $12 \pm 4$ \\
\hline \multicolumn{2}{|l|}{ At the time of terminal cardiovascular collapse } \\
\hline Base deficit (mmol/L) & $-5.1 \pm 8$ \\
\hline Arterial lactate (mmol/L) & $5.4 \pm 4.8$ \\
\hline Hemoglobin (mg/dl) & $9.6 \pm 1.9$ \\
\hline Partial arterial carbon dioxide tension $(\mathrm{mmHg})$ & $52 \pm 30$ \\
\hline Plethysmographic oxygen saturation (\%) & $77 \pm 16$ \\
\hline Body temperature $\left({ }^{\circ} \mathrm{C}\right)$ & $36.9 \pm 1.8$ \\
\hline Heart rate (beats/min) & $88 \pm 24$ \\
\hline Central venous pressure $(\mathrm{mmHg})$ & $14 \pm 7$ \\
\hline Catecholamine infusion (n (\%)) & $84(60)$ \\
\hline Vasopressin infusion ( $(\%))$ & $6(4.3)$ \\
\hline Levosimendan infusion ( $(\%))$ & $5(3.6)$ \\
\hline \multicolumn{2}{|l|}{ Cause of death } \\
\hline Cardiovascular failure $(n(\%))$ & $84(60)$ \\
\hline Multiple organ dysfunction (n (\%)) & $29(20.7)$ \\
\hline Respiratory failure (n (\%)) & $15(10.7)$ \\
\hline Severe ischemic encephalopathy ( $(\%))$ & $12(8.6)$ \\
\hline
\end{tabular}

Data are presented as mean values $\pm S D$, if not otherwise indicated.

cardiovascular collapse exhibited lower arterial blood pressure at terminal cardiovascular collapse than patients without sepsis. The incidences of comorbid conditions (congestive heart failure, $8.8 \%$ versus $22.6 \%, P=0.09$; left main stem stenosis, $8.8 \%$ versus $18.9 \%, P=0.2$; severe valvular aortic stenosis, $2.9 \%$ versus $1.9 \%, P=0.57$ ) and acute right heart failure $(17.6 \%$ versus $15.1 \%, P=0.79)$, as well as exposition to sedatives and/or opioids during the terminal phase $(88.2 \%$ versus $78.3 \%, P=0.32)$, did not differ between patients with sepsis and those without sepsis. No differences in arterial blood pressure at terminal cardiovascular collapse between the remaining subgroups were observed (Table 3).

Patients exposed to sedatives and/or opioids at the time of terminal cardiovascular collapse had lower 
Table 3 Arterial blood pressures at terminal cardiovascular collapse

\begin{tabular}{|c|c|c|c|c|c|c|c|c|c|}
\hline \multirow[b]{3}{*}{ Pre-defined risk factors } & \multirow{2}{*}{\multicolumn{3}{|c|}{$\frac{\text { Systolic arterial blood pressure }}{\text { (Total population, } 47 \pm 17 \text { ) }}$}} & \multirow{2}{*}{\multicolumn{3}{|c|}{$\frac{\text { Mean arterial blood pressure }}{\text { (Total population, } 35 \pm 11 \text { ) }}$}} & \multirow{2}{*}{\multicolumn{3}{|c|}{$\frac{\text { Diastolic arterial blood pressure }}{\text { (Total population, } 29 \pm 9 \text { ) }}$}} \\
\hline & & & & & & & & & \\
\hline & Risk factor & No risk factor & $P$-value & Risk factor & No risk factor & $P$-value & Risk factor & No risk factor & $P$-value \\
\hline Age $>65$ yr $(n=103)$ & $47 \pm 17$ & $47 \pm 18$ & 0.99 & $35 \pm 11$ & $34 \pm 12$ & 0.64 & $29 \pm 9$ & $28 \pm 10$ & 0.61 \\
\hline Age $>75$ yr $(n=66)$ & $50 \pm 17$ & $45 \pm 17$ & 0.06 & $37 \pm 10$ & $33 \pm 11$ & 0.05 & $30 \pm 9$ & $28 \pm 10$ & 0.16 \\
\hline Cardiac surgery $(n=10)$ & $51 \pm 19$ & $47 \pm 17$ & 0.53 & $35 \pm 12$ & $35 \pm 11$ & 0.95 & $27 \pm 11$ & $29 \pm 9$ & 0.69 \\
\hline $\begin{array}{l}\text { Chronic arterial hypertension } \\
(n=78)\end{array}$ & $49 \pm 17$ & $45 \pm 17$ & 0.12 & $36 \pm 11$ & $33 \pm 10$ & 0.18 & $29 \pm 10$ & $28 \pm 9$ & 0.29 \\
\hline Congestive heart failure $(n=27)$ & $53 \pm 20$ & $46 \pm 16$ & 0.06 & $39 \pm 13$ & $34 \pm 10$ & $0.04^{\mathrm{a}}$ & $31 \pm 12$ & $28 \pm 8$ & 0.17 \\
\hline Coronary artery disease $(n=57)$ & $48 \pm 18$ & $47 \pm 17$ & 0.76 & $36 \pm 12$ & $34 \pm 10$ & 0.34 & $30 \pm 10$ & $28 \pm 8$ & 0.27 \\
\hline Diabetes mellitus $(n=26)$ & $47 \pm 17$ & $48 \pm 17$ & 0.81 & $35 \pm 11$ & $35 \pm 11$ & 0.98 & $29 \pm 9$ & $28 \pm 9$ & 0.71 \\
\hline Left main stem stenosis $(n=23)$ & $53 \pm 16$ & $46 \pm 17$ & 0.08 & $39 \pm 11$ & $34 \pm 11$ & $0.03^{\mathrm{a}}$ & $32 \pm 10$ & $28 \pm 9$ & 0.07 \\
\hline $\operatorname{PAOD}^{\mathrm{b}}(n=15)$ & $43 \pm 17$ & $48 \pm 17$ & 0.34 & $33 \pm 10$ & $35 \pm 11$ & 0.63 & $27 \pm 8$ & $29 \pm 9$ & 0.62 \\
\hline Acute right heart failure $(n=22)$ & $53 \pm 20$ & $46 \pm 16$ & 0.1 & $39 \pm 13$ & $34 \pm 10$ & $0.03^{\mathrm{a}}$ & $32 \pm 11$ & $28 \pm 9$ & 0.06 \\
\hline Sepsis $(n=34)$ & $42 \pm 12$ & $49 \pm 18$ & $0.005^{\mathrm{a}}$ & $30 \pm 8$ & $36 \pm 11$ & $0.001^{\mathrm{a}}$ & $25 \pm 8$ & $30 \pm 9$ & $0.02^{\mathrm{a}}$ \\
\hline Severe aortic stenosis $(n=3)$ & $60 \pm 20$ & $47 \pm 17$ & 0.2 & $46 \pm 12$ & $34 \pm 11$ & 0.08 & $36 \pm 10$ & $28 \pm 9$ & 0.16 \\
\hline Shock $(n=87)$ & $48 \pm 17$ & $47 \pm 17$ & 0.73 & $35 \pm 11$ & $34 \pm 10$ & 0.36 & $29 \pm 10$ & $27 \pm 8$ & 0.14 \\
\hline
\end{tabular}

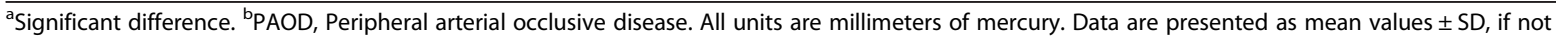
indicated otherwise.

systolic $(46 \pm 17 \mathrm{mmHg}$ versus $53 \pm 15 \mathrm{mmHg} ; P=0.048)$ and mean arterial blood pressures $(34 \pm 11 \mathrm{mmHg}$ versus $39 \pm 10 \mathrm{mmHg} ; P=0.03)$ than patients without sedative and/or opioid administration. The correlation analysis revealed a significant indirect relationship (Table 4). No significant correlation was observed between the arterial blood pressure at terminal cardiovascular collapse and other cofactors investigated (Table 4). There were also no relationships between the serum creatine kinase $\mathrm{MB}$ and arterial lactate levels during the last 24 hours with the mean arterial blood pressure at laboratory measurement or the arterial blood pressure at terminal cardiovascular collapse (Additional file 1: Figure S1).

\section{Discussion}

In this retrospective cohort study including 140 critically ill patients, the mean systolic, mean and diastolic arterial blood pressures associated with terminal cardiovascular collapse were 47,35 and $29 \mathrm{mmHg}$, respectively. Patients with congestive heart failure, left main stem stenosis, acute right heart failure or severe valvular aortic stenosis exhibited higher arterial blood pressures at terminal cardiovascular collapse than patients without these risk factors. Patients with sepsis had lower arterial blood pressures than patients without sepsis. Similarly, systolic and mean arterial blood pressures were lower in patients exposed to sedatives and/or opioids at the time of terminal cardiovascular collapse.

In our analysis, we identified the arterial blood pressure associated with terminal cardiovascular collapse as indicated by an abrupt and exponential drop in heart rate. It is conceivable that, at these low arterial blood pressure levels, coronary perfusion was reduced to such an extent that global myocardial hypoperfusion and ischemia occurred. As we did not measure coronary blood flow in our population, no association between the arterial blood pressure at terminal cardiovascular collapse and the lower threshold of coronary autoregulation can be made. From a physiologic perspective, it is likely that terminal cardiovascular collapse occurs at arterial blood pressures well below the lower coronary autoregulation limit and that adverse events such as coronary or endorgan hypoperfusion occur at higher arterial blood pressures than the ones identified in this study [17]. Therefore, the arterial blood pressures associated with terminal cardiovascular collapse should by no means implicate safe values or be regarded as a resuscitation endpoint. They should instead be considered as the lowest end of cardiovascular regulation in a mixed critically ill patient population. Even when transiently applying the strategy of permissive hypotension, these arterial blood pressure levels should never be reached or tolerated, but rather an individual safety limit (for example, one or two standard deviations) above these arterial blood pressure levels should be maintained.

Five subgroups presented with arterial blood pressures at terminal cardiovascular collapse that were different from those in the remaining population. The respective mean arterial blood pressure levels differed by approximately $5 \mathrm{mmHg}$ between patients with and those without acute right heart failure. This finding is in line with clinical observations that patients with acute right heart 


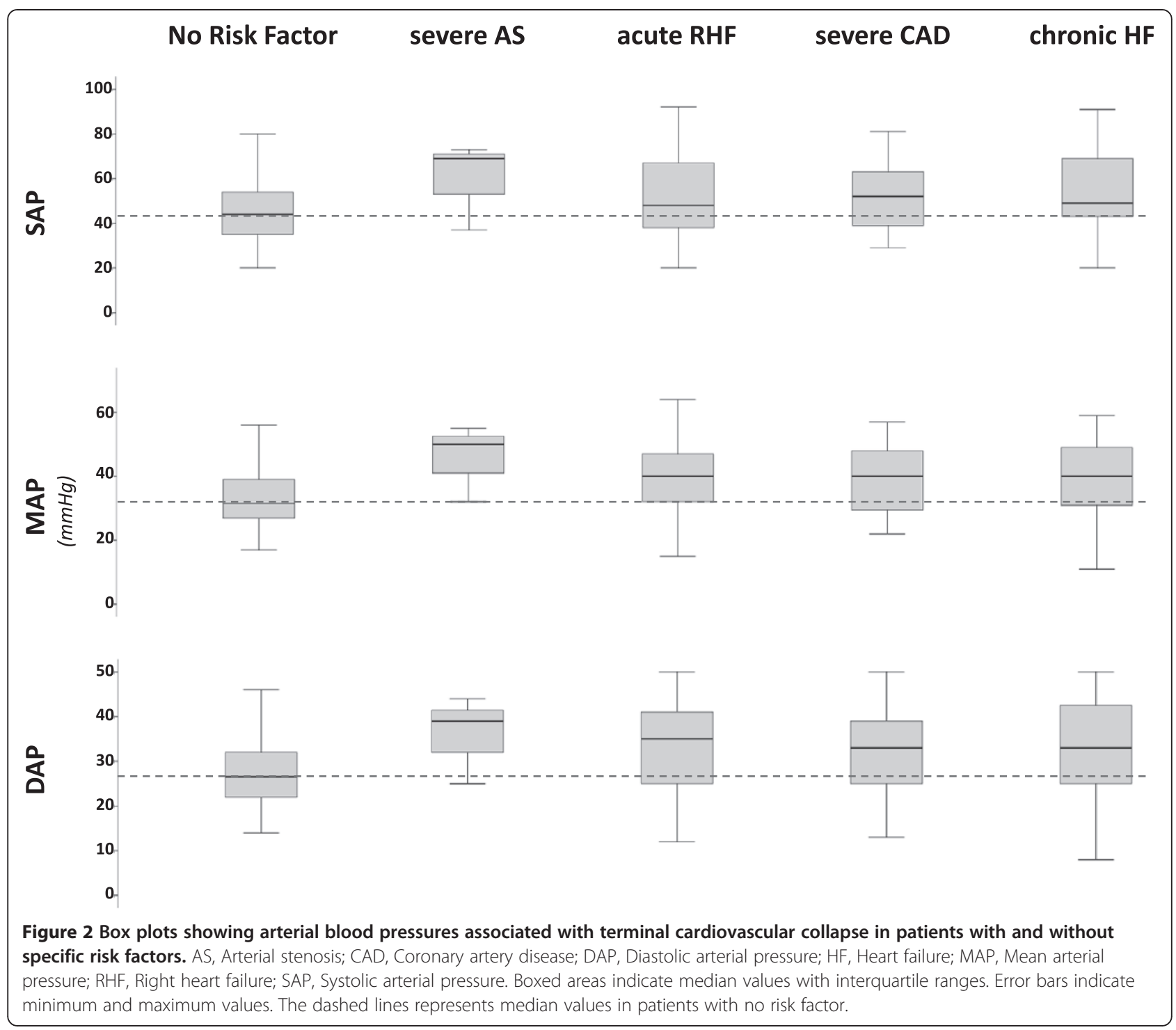

Table 4 Bivariate correlations between arterial blood pressures at terminal cardiovascular collapse and predefined cofactors $^{\mathrm{a}}$

\begin{tabular}{|c|c|c|c|c|c|c|}
\hline \multirow[t]{2}{*}{ Cofactor } & \multicolumn{2}{|c|}{ Systolic arterial blood pressure } & \multicolumn{2}{|c|}{ Mean arterial blood pressure } & \multicolumn{2}{|c|}{ Diastolic arterial blood pressure } \\
\hline & Pearson coefficient & $P$-value & Pearson coefficient & $P$-value & Pearson coefficient & $P$-value \\
\hline Age (yr) & 0.1 & 0.24 & 0.11 & 0.2 & 0.07 & 0.38 \\
\hline $\mathrm{SpO}_{2}(\%)$ & -0.06 & 0.54 & 0.02 & 0.82 & 0.05 & 0.63 \\
\hline Temperature $\left({ }^{\circ} \mathrm{C}\right)$ & -0.08 & 0.41 & -0.09 & 0.31 & -0.13 & 0.17 \\
\hline Hemoglobin (g/dl) & 0.03 & 0.69 & 0.04 & 0.69 & $<0.01$ & 0.99 \\
\hline $\mathrm{PaCO}_{2}(\mathrm{mmHg})$ & 0.15 & 0.08 & 0.08 & 0.38 & 0.03 & 0.7 \\
\hline SOFA score (points) & -0.04 & 0.61 & 0.06 & 0.48 & 0.14 & 0.11 \\
\hline Sedative and/or opioid exposure & -0.17 & $0.048^{\mathrm{b}}$ & -0.185 & $0.03^{b}$ & -0.16 & 0.06 \\
\hline
\end{tabular}

${ }^{\mathrm{a}} \mathrm{PaCO}_{2}$, partial carbon dioxide pressure; SOFA, Sequential Organ Failure Assessment; $\mathrm{SpO}_{2}$, plethysmographic oxygen saturation. ${ }^{\mathrm{b}}$ Significant correlation. 
failure require an adequate perfusion pressure to maintain right ventricular function $[18,19]$. Patients with congestive heart failure and left main stem stenosis exhibited mean arterial blood pressures at terminal cardiovascular collapse that were, on average, 3 to $7 \mathrm{mmHg}$ above those of patients without these risk factors. As three-fourths of patients with congestive heart failure had coronary artery disease and one-half of them from left main stem stenosis or an equivalent, the two subgroups are likely to share similar reasons for higher arterial pressure requirements. Higher arterial blood pressures are similarly required to maintain coronary blood flow in patients with significant coronary stenosis [20,21]. Although patients with severe valvular aortic stenosis exhibited the highest arterial blood pressures at terminal cardiovascular collapse in this cohort, comparisons with the remaining population failed to reach the significance level, owing to the small number of patients $(n=3)$. Also, in severe aortic stenosis, arterial blood pressure is a key determinant of coronary perfusion [22,23], which explains why these patients required arterial blood pressures 8 to $13 \mathrm{mmHg}$ higher than patients without severe aortic stenosis. Patients with sepsis experienced terminal cardiovascular collapse only when arterial blood pressures were 5 to $7 \mathrm{mmHg}$ lower than in the remaining population. This observation is striking and cannot be explained by differences in comorbid conditions or the incidence of acute right heart failure in these patients. It may be hypothesized that one reason for this observation is that sepsis patients had higher cardiac output levels than patients without sepsis and could thus tolerate lower arterial blood pressures. However, our study cannot prove this hypothesis, because cardiac output was not measured in any study patient during the terminal phase. Although no further differences in arterial blood pressures at terminal cardiovascular collapse could be identified, our analysis may have included too few patients to uncover minor differences between other subgroups and some of the observed variations between subgroups may have equally resulted from small patient numbers in each group.

Another interesting finding of this analysis is that patients receiving sedatives and/or opioids during the terminal phase experienced terminal cardiovascular collapse at significantly lower arterial blood pressures than patients who were not exposed to such drugs. This observation may have important practical implications because terminal cardiovascular collapse in awake patients or patients not exposed to sedative agents or opioids may occur at relevantly higher arterial blood pressure levels (5 to $8 \mathrm{mmHg}$ on average) than in sedated patients. Other investigators have postulated that sedative agents and opioids can reduce oxygen requirements and hence increase the body's tolerance to hypoxia and arterial hypotension [24], as may have been the case in our cohort. These results are in accordance with data showing that a $1 \mathrm{mg} / \mathrm{hr}$ increase of the benzodiazepine dose after withdrawal of life support increased the time until death by 13 minutes [25]. Further correlation analyses between arterial blood pressure at terminal cardiovascular collapse and various other cofactors did not reveal significant relationships. Several reasons may account for this unexpected finding. First, 140 patients may still have been too small a study population to detect some correlations. Second, cofactors that were not included in our correlation analysis may play a relevant role. For example, it is likely that the individual hemodynamic management and duration of preceding arterial hypotension, particularly the time spent with arterial blood pressures below the lower coronary autoregulation threshold, may affect the arterial blood pressure at terminal cardiovascular collapse.

When interpreting the results of this study, additional limitations need to be noted. This was a retrospective analysis and may thus contain methodological weaknesses such as missing values and heterogeneous datasets. A relevant selection bias may have been introduced into the analysis in that $20.9 \%$ of patients who originally met the inclusion criteria were excluded because their arterial blood pressure at terminal cardiovascular collapse could not be determined or because their terminal cardiovascular collapse was caused by rhythmologic pathologies. Although we failed to identify an arterial blood pressure at which rhythmologic pathologies occurred in patients excluded from this analysis (data not shown), it is possible that such terminal rhythm disturbances might have been the consequence of coronary hypoperfusion and that a delicate group of patients exclusively sensitive to low arterial blood pressure levels was excluded from our analysis.

\section{Conclusions}

The arterial blood pressure associated with terminal cardiovascular collapse in critically ill patients was very low and varied with individual comorbid conditions (for example, congestive heart failure, left main stem stenosis, severe valvular aortic stenosis, acute right heart failure), drug exposure (for example, sedatives and/or opioids) and the type of acute illness (for example, sepsis).

\section{Key messages}

- The arterial blood pressure associated with terminal cardiovascular collapse in critically ill patients was very low at $47 \pm 12 \mathrm{mmHg}$ systolic, $35 \pm 11 \mathrm{mmHg}$ mean and $29 \pm 9 \mathrm{mmHg}$ diastolic arterial blood pressure.

- Relevant exceptions were patients with congestive heart failure, left main stem stenosis, severe valvular aortic stenosis or acute right heart failure. 
- Sepsis patients and patients receiving sedatives and/ or opioids exhibited even lower arterial blood pressures at terminal cardiovascular collapse.

\section{Additional file}

Additional file 1: Figure S1. Arterial lactate levels and critical MAP values in patients stratified into different MAP levels at last lactate measurement ( $242 \pm 280$ minutes before cardiovascular collapse).

\section{Abbreviations}

AS: Arterial stenosis; CAD: Coronary artery disease; DAP: Diastolic arterial pressure; HF: Heart failure; ICU: Intensive care unit; MAP: Mean arterial pressure; $\mathrm{PaCO}_{2}$ : Partial carbon dioxide pressure; PAOD: peripheral arterial occlusive disease; RHF: Right heart failure; $\mathrm{SpO}_{2}$ : Plethysmographic oxygen saturation; SAP: Systolic arterial pressure; SAPS: Simplified Acute Physiology Score; SOFA: Sequential Organ Failure Assessment.

\section{Competing interests}

The authors declare that they have no competing interests.

\section{Authors' contributions}

$A B$ and MWD designed the study, collected data, interpreted the results, drafted the manuscript and revised it for important intellectual content. AK, IGM and DD collected data, interpreted the results and revised the manuscript for important intellectual content. OB collected data and revised the manuscript for important intellectual content. All authors read and approved the final manuscript.

\section{Author details}

${ }^{1}$ Department of Anesthesiology, Perioperative Care and Intensive Care Medicine, University Hospital Salzburg and Paracelsus Private Medical University, Müllner Hauptstrasse 48, 5020 Salzburg, Austria. ${ }^{2}$ Department of Emergency and Critical Care Medicine, Central State University Hospital, Marx Street, Ulaanbaatar, Mongolia.

Received: 6 August 2014 Accepted: 11 December 2014

Published online: 19 December 2014

\section{References}

1. Vincent JL, De Backer D: Circulatory shock. N Engl J Med 2013, 369:1726-1734.

2. Lipcsey M, Castegren M, Bellomo R: Hemodynamic management of septic shock. Minerva Anestesiol, in press.

3. Dellinger RP, Levy MM, Rhodes A, Annane D, Gerlach H, Opal SM, Sevransky JE, Sprung $C L$, Douglas IS, Jaeschke R, Osborn TM, Nunnally ME, Townsend SR, Reinhart K, Kleinpell RM, Angus DC, Deutschman CS, Machado FR, Rubenfeld GD, Webb S, Beale RJ, Vincent JL, Moreno R, Surviving Sepsis Campaign Guidelines Committee including The Pediatric Subgroup: Surviving Sepsis Campaign: international guidelines for management of severe sepsis and septic shock, 2012. Intensive Care Med 2013, 39:165-228.

4. Pinsky MR: Targets for resuscitation from shock. Minerva Anestesiol 2003, 69:237-244.

5. Waechter J, Kumar A, Lapinsky SE, Marshall J, Dodek P, Arabi Y, Parrillo JE, Dellinger RP, Garland A, Cooperative Antimicrobial Therapy of Septic Shock Database Research Group: Interaction between fluids and vasoactive agents on mortality in septic shock: a multicenter, observational study. Crit Care Med 2014, 42:2158-2168

6. Sperry JL, Minei JP, Frankel HL, West MA, Harbrecht BG, Moore EE, Maier RV, Nirula R: Early use of vasopressors after injury: caution before constriction. J Trauma 2008, 64:9-14

7. Subramanian S, Yilmaz M, Rehman A, Hubmayr RD, Afessa B, Gajic O: Liberal vs. conservative vasopressor use to maintain mean arterial blood pressure during resuscitation of septic shock: an observational study. Intensive Care Med 2008, 34:157-162.

8. Beck V, Chateau D, Bryson GL, Pisipati A, Zanotti S, Parrillo JE, Kumar A Cooperative Antimicrobial Therapy of Septic Shock (CATSS) Database Research Group: Timing of vasopressor initiation and mortality in septic shock: a cohort study. Crit Care 2014, 18:R97.
9. Curry N, Davis PW: What's new in resuscitation strategies for the patient with multiple trauma? Injury 2012, 43:1021-1028.

10. Dünser MW, Takala J, Brunauer A, Bakker J: Re-thinking resuscitation: leaving blood pressure cosmetics behind and moving forward to permissive hypotension and a tissue perfusion-based approach. Crit Care 2013, 17:326.

11. Raab $H$, Lindner $\mathrm{KH}$, Wenzel V: Preventing cardiac arrest during hemorrhagic shock with vasopressin. Crit Care Med 2008, 36:474-480

12. Mäkivirta A, Koski E, Kari A, Sukuvaara T: The median filter as a preprocessor for a patient monitor limit alarm system in intensive care. Comput Methods Programs Biomed 1991, 34:139-144.

13. Le Gall JR, Lemeshow S, Saulnier F: A new Simplified Acute Physiology Score (SAPS II) based on a European/North American multicenter study. JAMA 1993, 270:2957-2963. A published erratum appears in JAMA 1994, 271:1321.

14. Metnitz PG, Moreno RP, Almeida E, Jordan B, Bauer P, Campos RA, lapichino G, Edbrooke D, Capuzzo M, Le Gall JR, SAPS 3 Investigators: SAPS 3-from evaluation of the patient to evaluation of the intensive care unit. Part 1: objectives, methods and cohort description. Intensive Care Med 2005, 31:1336-1344.

15. Vincent $J$, de Mendonça A, Cantraine F, Moreno R, Takala J, Suter PM, Sprung $\mathrm{CL}$, Colardyn F, Blecher S: Use of the SOFA score to assess the incidence of organ dysfunction/failure in intensive care units: results of a multicenter, prospective study. Working group on "sepsis-related problems" of the European Society of Intensive Care Medicine. Crit Care Med 1998, 26:1793-1800.

16. Levy MM, Fink MP, Marshall JC, Abraham E, Angus D, Cook D, Cohen J, Opal SM, Vincent JL, Ramsay G, SCCM/ESICM/ACCP/ATS/SIS: 2001 SCCM/ESICM/ACCP/ ATS/SIS International Sepsis Definitions Conference. Crit Care Med 2003, 31:1250-1256.

17. Baumgart D, Haude M, Liu F, Ge J, Goerge G, Erbel R: Current concepts of coronary flow reserve for clinical decision making during cardiac catheterization. Am Heart J 1998, 136:136-149.

18. Rich S, Gubin S, Hart K: The effects of phenylephrine on right ventricular performance in patients with pulmonary hypertension. Chest 1990, 98:1102-1106

19. Vlahakes GJ, Turley K, Hoffman Jl: The pathophysiology of failure in acute right ventricular hypertension: hemodynamic and biochemical correlations. Circulation 1981, 63:87-95.

20. Meier J, Kleen M, Messmer K: A computer model of fractal myocardial perfusion heterogeneity to elucidate mechanisms of changes in critical coronary stenosis and hypotension. Bull Math Biol 2004, 66:1155-1171.

21. Hickey RF, Verrier ED, Baer RW, Vlahakes GJ, Fein G, Hoffman J: A canine model of acute coronary artery stenosis: effects of deliberate hypotension. Anesthesiology 1983, 59:226-236

22. Kirkeeide RL, Gould KL, Parsel L: Assessment of coronary stenoses by myocardial perfusion imaging during pharmacologic coronary vasodilation. VII. Validation of coronary flow reserve as a single integrated functional measure of stenosis severity reflecting all its geometric dimensions. J Am Coll Cardiol 1986, 7:103-113.

23. Hinchman DA, Otto CM: Valvular disease in the elderly. Cardiol Clin 1999 $17: 137-158$

24. Bakker J, Jansen TC, Lima A, Kompanje EJ: Why opioids and sedatives may prolong life rather than hasten death after ventilator withdrawal in critically ill patients. Am J Hosp Palliat Care 2008, 25:152-154

25. Chan JD, Treece PD, Engelberg RA, Crowley L, Rubenfeld GD, Steinberg KP, Curtis JR: Narcotic and benzodiazepline use after withdrawal of life support: association with time to death? Chest 2004, 126:286-293. 